\title{
Implante Percutâneo de Válvula Aórtica Auxiliada pela Oxigenação por Membrana Extracorpórea para Tratamento de Estenose Aórtica com Choque Cardiogênico
}

\author{
Transcatheter Aortic Valve Implantation Assisted by Extracorporeal Membrane Oxygenation for the Treatment \\ of Aortic Stenosis with Cardiogenic Shock
}

Gangping Huang, ${ }^{1}$ Huaidong Chen, ${ }^{1}$ Weimin Zhang, ${ }^{10}$ Fan $\mathrm{He}^{1 \text { (]) }}$

Departamento de Cirurgia Cardíaca, Hospital Sir Run Run Shaw, Escola de Medicina, Universidade de Zhejiang, ${ }^{1}$ Zhejiang - China

\section{Introdução}

A estenose aórtica é uma doença valvular cardíaca comum geralmente causada por uma doença degenerativa da válvula aórtica nos idosos. ${ }^{1}$ A estenose aórtica obstrui o fluxo para a frente do ventrículo esquerdo até a aorta, levando a um diferencial de pressão entre o ventrículo esquerdo e a aorta e o aumento da pressão do ventrículo esquerdo secundário à hipertrofia do ventrículo esquerdo. À medida que a doença avança, ela leva à disfunção diastólica e sistólica do ventrículo esquerdo e à isquemia miocárdica devido à diminuição do fluxo sanguíneo coronário. Entretanto, o choque cardiogênico secundário à estenose aórtica é uma das complicações mais sérias e tem um alto índice de mortalidade devido a seu efeito terapêutico limitado. ${ }^{2}$ Desde seu início em 2002, o implante percutâneo da válvula aórtica (TAVI) se tornou o tratamento de escolha para pacientes idosos com estenose aórtica grave e alto risco cirúrgico devido a suas vantagens de ser minimamente invasivo, circulação não extracorpórea, e boa eficácia de meio e longo prazo. ${ }^{3-5}$ Com o desenvolvimento da tecnologia dos dispositivos e sistemas de transmissão de baixa resistência, o TAVI demonstrou recentemente ser tão eficiente do que a cirurgia tradicional, mesmo em pacientes de risco médio. ${ }^{6}$ Entretanto, para pacientes com histórico médico longo, ele diminuiu significativamente a fração de ejeção cardíaca (FE), choque cardiogênico, doença da válvula aórtica descompensada e complicações graves, tais como a instabilidade hemodinâmica intraoperatória e arritmia maligna, ainda existem durante a cirurgia com TAVI, aumentando significativamente o risco de TAVI. Neste estudo, relata-se um caso de estenose aórtica grave complicada por choque cardiogênico que foi tratado com sucesso com TAVI auxiliado pela oxigenação por membrana extracorpórea.

\section{Palavras-chave}

Oxigenação por Membrana Extracorpórea; Estenose da Valva Aórtica; Choque cardiogênico.

\author{
Correspondência: Fan He • \\ Departamento de Cirurgia Cardíaca, Hospital Sir Run Run Shaw, Escola de \\ Medicina, Universidade de Zhejiang - East Qingchun Road 3th, Hangzhou, \\ Hangzhou 310016 - China \\ E-mail: hefan8917@zju.edu.cn \\ Artigo recebido em 23/12/2020, revisado em 28/04/2021, aceito em \\ $28 / 04 / 2021$
}

DOI: https://doi.org/10.36660/abc.20201358

\section{Relato de Caso}

Uma paciente de 64 anos, do sexo feminino, foi hospitalizada devido a "pressão no peito e fadiga por mais de 2 meses e agravamento por 3 dias". Ela tinha história prévia de colecistectomia por 10 anos. O exame físico na admissão indicou uma temperatura de $36,8{ }^{\circ} \mathrm{C}$, 18 respirações/ minuto, pulso de 46 batimentos/minuto, e pressão arterial de 136/92 mmHg. A paciente tinha ortopneia. Os sons pulmonares de ambos os lados eram grossos, e podia ser ouvido um ronco úmido. O sopro sistólico podia ser ouvido na auscultação da válvula aórtica e havia um ligeiro linfedema em ambas as pernas. Exames laboratoriais mostraram peptídeo natriurético pró-cerebral N-terminal (NT-proBNP)>25000 pg/ $\mathrm{mL}$, Troponina I (Tnl) 0,12 $\mu \mathrm{g} / \mathrm{L}$. Os índices de função renal e hepática também aumentaram significativamente. O ecocardiograma sugeriu estenose aórtica grave com insuficiência leve, o gradiente de pressão sistólica máximo era de $130 \mathrm{mmHg}$ e o ventrículo esquerdo foi significativamente aumentado (LVIDd:58,3mm) com disfunção diastólica e sistólica. A FE foi medida em 23,5\% pelo método biplanar (Figura 1). O TC do tórax demonstrou exsudato pleural duplo, edema intersticial pulmonar, efusão encapsulada de ambos os pulmões, com insuficiência pulmonar. O ECG dinâmico de 24 horas sugere o ritmo sinusal com batimentos prematuros atriais e ventriculares frequentes. Os resultados da aferição da pressão arterial ambulatorial demonstrou que a pressão arterial foi de 96/64 mmHg durante o dia, 98/65 mmHg durante o dia, e 93/62 mmHg à noite. A avaliação do TAVI por imagens dos pacientes, e os resultados demonstraram estenose aórtica típica com malformação bicúspide (tipo 0) e calcificação moderada (Figura 2). Os diagnósticos foram estenose aórtica e choque cardiogênico com uma função cardíaca de classificação NYHA IV. O paciente recebeu cardiotônicos, diuréticos, ventilação não invasiva adjuvante, mas a insuficiência cardíaca e os sintomas respiratórios não melhoraram. Considerando que o paciente tinha alto risco de choque cardiogênico e insuficiência cardíaca causada pela estenose aórtica, não houve condições de realização de uma cirurgia aberta. O risco da cirurgia TAVI também era muito alto. Então, depois de uma consulta multidisciplinar, foi proposto um plano de tratamento com cirurgia TAVI auxiliada por ECMO. A intubação traqueal foi inserida sob anestesia geral. $\mathrm{O}$ cateter guia $6 \mathrm{~F}$ estava intravesical à veia jugular direita, e, em seguida, o marca-passo temporário foi inserido no ventrículo direito pelo cateter guia. A artéria femoral direita foi puncionada com uma agulha de microperfuração $4 \mathrm{~F}$, o cateter, de $6 \mathrm{~F}$ a 11F, foi utilizado para expandir, e 2 dispositivos de 


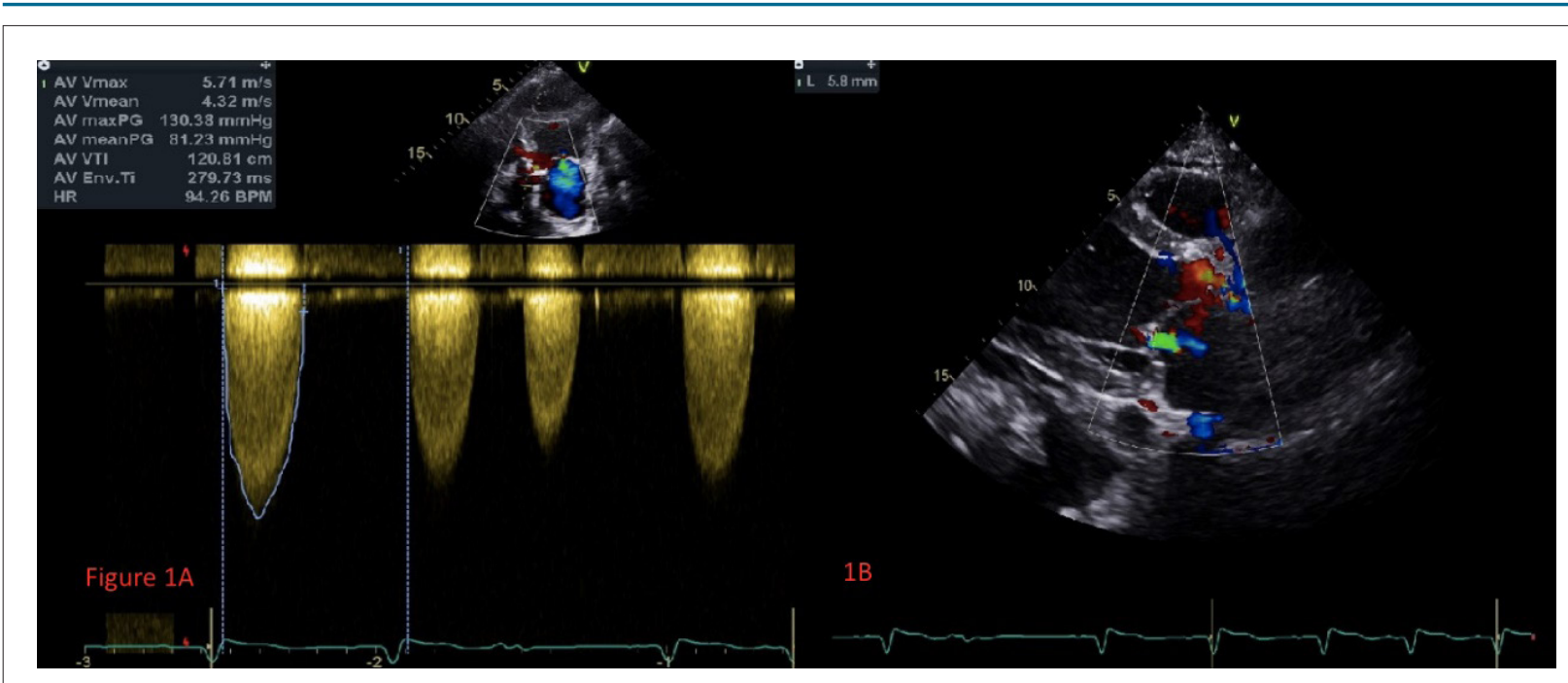

Figura 1 - $A$ e B) 0 ecocardiograma pré-operatório indicado indicou que o gradiente de pressão e a velocidade da válvula aórtica aumentaram, a abertura da válvula aórtica se calcificou, e ocorreu estenose aórtica grave.

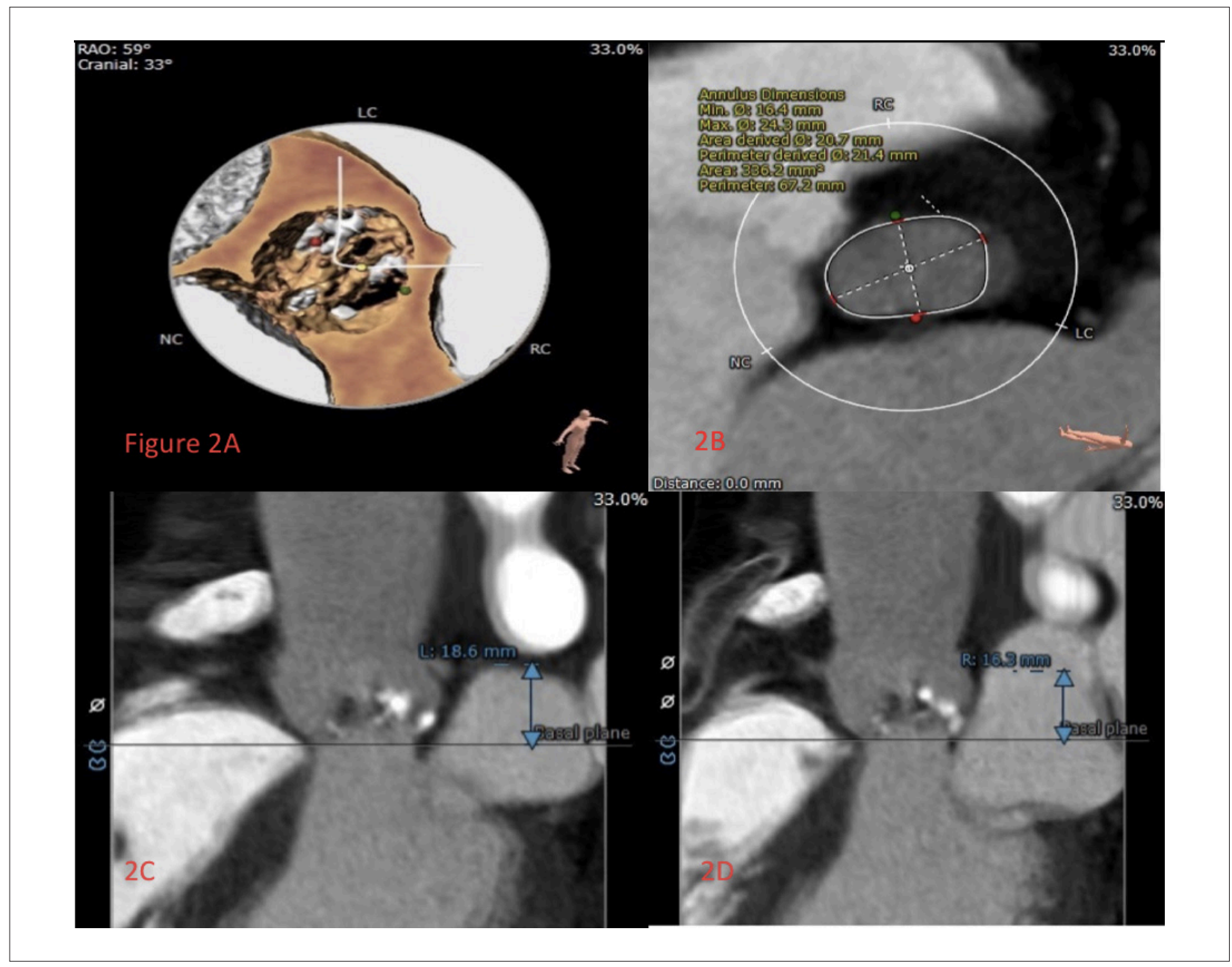

Figura 2 - Reconstrução tridimensional da válvula aórtica (2A), diâmetro anular aórtico (2B), altura do óstio coronário esquerdo (2C), e óstio coronário direito (2D). 


\section{Relato de Caso}

fechamento Perclose Proglide (Abbott Vascular, Minneapolis, $\mathrm{MN}, \mathrm{EUA}$ ) foram inseridos para aplicação em standby, e, em seguida, o tubo 18F foi inserido. A oxigenação por membrana extracorpórea venoarterial (VA-ECMO) foi realizada inserindo-se uma cânula arterial 16F e uma cânula venosa 22F na artéria femoral esquerda e na veia femoral, respectivamente. O fluxo assistido por circulação era de 2,7 L/min, e a pressão arterial foi mantida em aproximadamente $120 \mathrm{mmHg}$. Durante a operação, é necessário garantir que a posição das cânulas arterial e venosa está em boa condição para evitar puxões, dobras, deslocamento e prolapso. Também é importante observar a cor do sangue e a tensão do lúmen, se há instabilidade no lúmen, se há coágulos sanguíneos, e se a hemodinâmica está estável durante o período de circulação assistida. Um cateter foi colocado no ventrículo esquerdo via o cateter guia da artéria radial esquerda e apresentou pressão de VE de 167/25 mmHg e pressão arterial aórtica de 100/77 $\mathrm{mmHg}$. O fio guia foi enviado ao ventrículo esquerdo pelo cateter guia $18 \mathrm{~F}$ e, em seguida, colocado no balão Numed $18 \mathrm{~mm}$ e expandido após definir o ritmo cardíaco temporário em 180 batimentos/ min. Baseado nos dados medidos da reconstrução de TC, foi selecionada uma válvula Venus A-Valve de $23 \mathrm{~mm}$ (Venus MedTech, Hangzhou, China). A válvula foi liberada em um posicionamento preciso e ritmo cardíaco temporário de 160 batimentos/min. Os resultados indicaram que a forma e a posição da válvula eram boas, e a angiografia indicou uma pequena quantidade de vazamento perivascular (Figura 3).

Após o procedimento, o paciente, confiante no suporte total do ECMO e de fármacos vasoativos para manter a hemodinâmica, foi transferido para a unidade de terapia intensiva (UTI). A hemodinâmica do paciente estava estável e o ECMO foi retirado 20 horas após a cirurgia. Devido à atelectasia pré-operatória e ao edema pulmonar, a intubação traqueal foi extraída três dias após a operação. Os sintomas e sinais pós-operatórios do paciente melhoraram significativamente, os índices de NT-proBNP, Tnl, e de função hepática e renal diminuíram significativamente. O ecocardiograma pós-operatório indicou uma função valvular normal acompanhada de uma pequena quantidade de vazamento perivalvular. A velocidade do orifício e o gradiente de pressão foram reduzidos significativamente em comparação aos anteriores à cirurgia, e o FE aumentou para 66\% (Figura 4). Devido às condições graves do paciente antes da cirurgia, acamamento pós-operatório longo, desnutrição e influência de drogas, ocorreram sintomas pós-operatórios, tais como transtorno de consciência, infecção pulmonar, e trombose venosa intermuscular bilateral das extremidades. Pela melhoria do equilíbrio do ambiente interno, nutrição, exercícios funcionais anti-infecção e de reabilitação pós-operatório, o paciente finalmente recebeu a alta com sucesso.

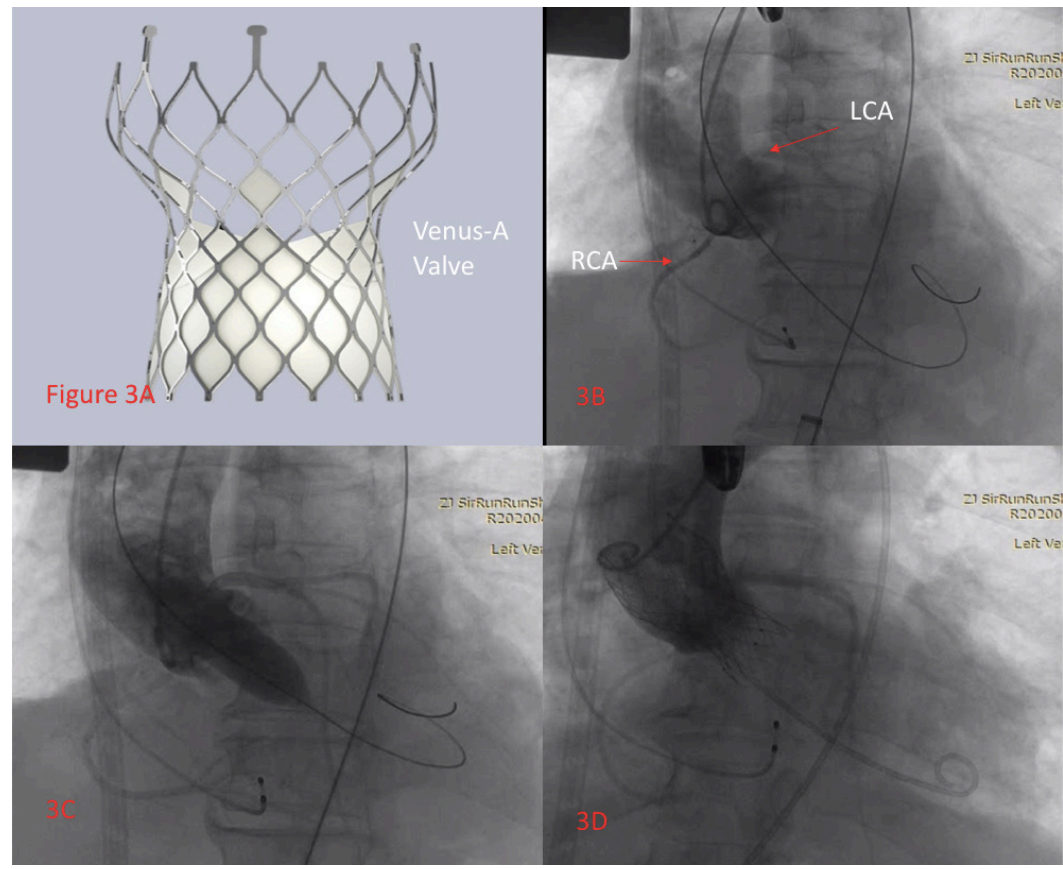

Figura 3 - A) A válvula utilizada era da China; B) Aortografia antes da dilatação do balão indicou que as artérias coronárias estavam bem desenvolvidas, e que havia estenose grave da válvula aórtica; C) Aortografia com o balão totalmente inflado mostrando a patência das artérias coronárias esquerda e direita; D) Aortografia final após a instalação da válvula e dilatação demonstrou um bom posicionamento da válvula e uma pequena quantidade de vazamento perivalvular. 


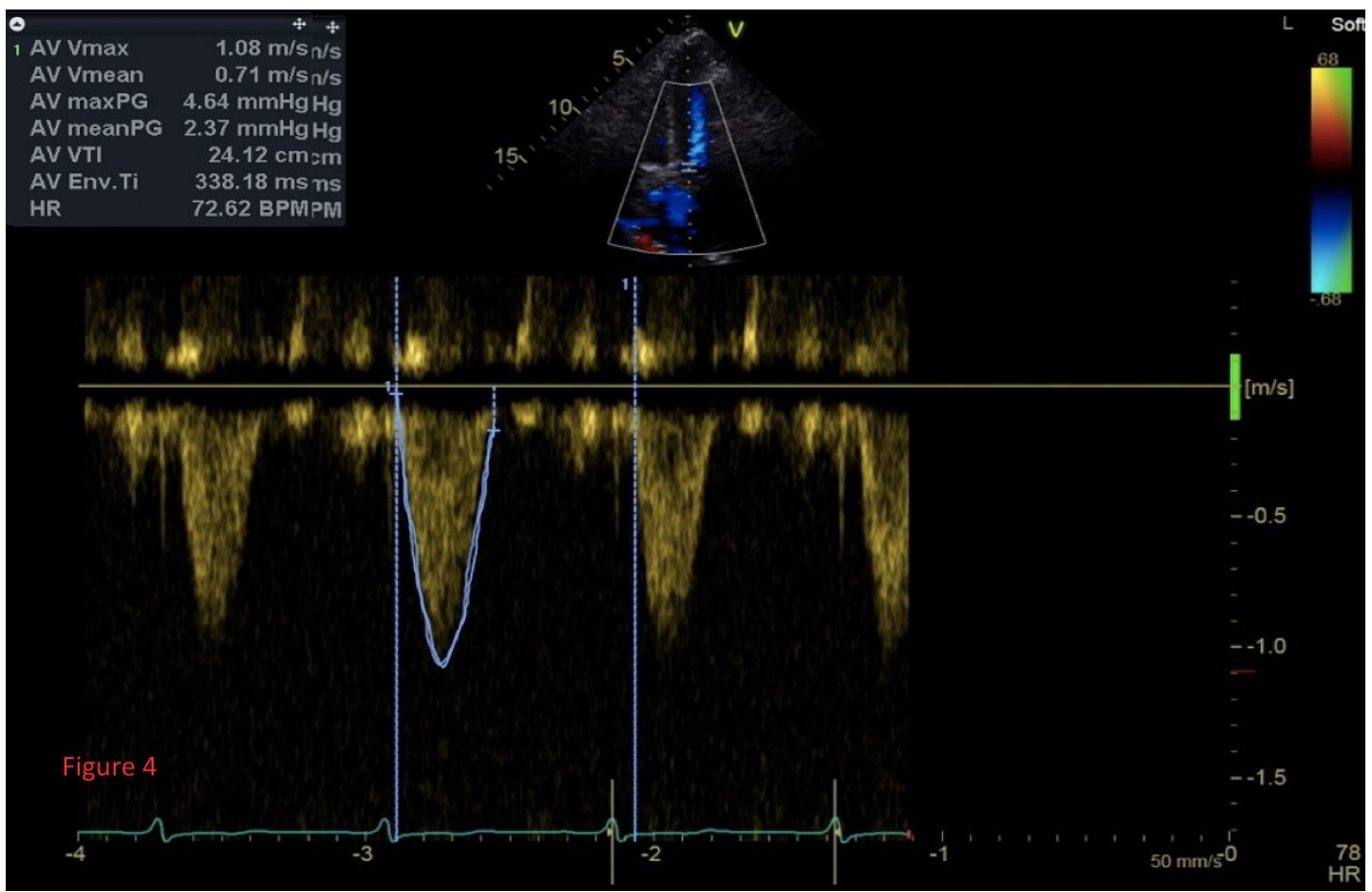

Figura 4 - 0 ecocardiograma pós-operatório indicado indicou que o gradiente de pressão e a velocidade da válvula aórtica melhoraram significativamente.

\section{Discussão}

TAVI é uma nova tecnologia para tratamento de doença aórtica. Depois de mais de uma década de desenvolvimento, o TAVI surgiu como uma opção de tratamento atraente e menos invasiva para a estenose aórtica grave, e é superior ao tratamento farmacológico para pacientes que não podem ser submetidos à cirurgia convencional. ${ }^{7,8} \mathrm{Com}$ avanços modernos em equipamentos, o TAVI demonstrou ser tão eficiente quanto a cirurgia tradicional em pacientes com risco intermediário. ${ }^{6}$ Entretanto, na prática clínica, alguns pacientes foram associados ao FE baixo, ventrículo esquerdo reduzido, choque cardiogênico, e outros sintomas antes da cirurgia, que, sem dúvidas, aumentaram o risco da cirurgia TAVI. Encontrar um tratamento seguro e eficiente para esses pacientes com risco muito alto é sempre um desafio para os cardiologistas. O ECMO é um dispositivo de suporte circulatório mecânico que tem sido usado para auxiliar o tratamento adjuvante do choque cardiogênico e ressuscitação cardiopulmonar causados por vários motivos nos últimos anos. ${ }^{9}$ Para pacientes com instabilidade hemodinâmica, o ECMO pode garantir fluxo sanguíneo e suprimento de oxigênio estáveis e, portanto, tratar a insuficiência cardíaca reversível com eficiência. ${ }^{10}$ Entretanto, para pacientes com estenose aórtica grave com choque cardiogênico, a experiência do ECMO no TAVI é limitada. Um estudo observacional ${ }^{11}$ examinou os resultados do TAVI transapical em pacientes com choque cardiogênico e detectou que a presença do choque cardiogênico aumentava significativamente a mortalidade 30 dias após o TAVI (19\% choque cardiogênico vs. 5\% sem choque cardiogênico; $\mathrm{p}=0,02)$. Entretanto, o índice de mortalidade do TAVI no grupo com choque cardiogênico ainda era mais baixo do após a substituição de válvula aórtica convencional (19\% vs. $26 \%$ ), sugerindo que o TAVI pode ser uma opção de tratamento viável para choque cardiogênico. O paciente deste estudo apresentou sintomas de choque cardiogênico grave após a admissão, tais como hipotensão e ortopneia, e tinha um alto risco, com classificação STS de 30,06. A cirurgia de troca de válvula aórtica tradicional tem um risco muito alto. Entretanto, a cirurgia TAVI nesse momento também aumente, sem dúvidas, o risco de instabilidade hemodinâmica intraoperatória, arritmia maligna e até mesmo morte súbita. Além disso, tratamentos conservadores, tais como cardiotônicos, diuréticos, e ventilação mecânica não invasiva não conseguiram melhorar os sintomas do paciente, podendo levar à morte. Portanto, se ocorrer o colapso circulatório, ou o paciente parecer ser intolerante ao TAVI, não se deve hesitar em usar o ECMO. De acordo com nossa experiência prática, o uso intraoperatório do ECMO garante a estabilização da hemodinâmica, permite, a expansão repetida da válvula aórtica doente sem arritmias malignas, tais como taquicardia supraventricular e fibrilação ventricular, reduz significativamente o risco do procedimento do TAVI, reduz significativamente a irritabilidade cardíaca intraoperatória possível em etapas fundamentais da cirurgia, tais como a expansão do balão na válvula aórtica e a liberação da válvula interveniente, e garante efetivamente a segurança da cirurgia em pacientes de alto risco. Ao mesmo tempo, para pacientes 
com estenose aórtica grave, a pressão arterial foi melhorada em níveis diferentes após a dilatação do balão assistida por ECMO, evitando o risco de colapso circulatório causado pela dilatação do balão em um estado não protegido desses pacientes, de forma que os pacientes possam se beneficiar ao máximo.

Entretanto, a incidência de complicações associadas ao ECMO (tais como isquemia de membros inferiores, acidente vascular cerebral, lesão vascular, lesão renal aguda, sangramento, e infecção) é tão alta que é essencial, para o uso eficiente e racional do ECMO por uma equipe de saúde experiente. ${ }^{12}$ Por outro lado, após a retirada bem-sucedida do ECMO, deve-se prestar mais atenção ao controle pósoperatório para melhorar os resultados pós-operatórios, tais como o uso de medicamento para insuficiência cardíaca, intervenção abrangente em comorbidades, reabilitação cardíaca prolongada, e acompanhamento ambulatorial próximo. O paciente deste estudo apresentou transtorno de consciência pós-operatório, e o TC não revelou sinais de infarto cerebral ou hemorragia cerebral, que poderiam ser causados pelo uso excessivo de sedativos. Assim, tomamos a decisão de interromper o uso de sedativos e administramos terapia neurotrófica. A alta antecipada da unidade de terapia intensiva pode reduzir a incidência de infecção cruzada e infecção iatrogênica após a operação. Além disso, exercícios de reabilitação pós-operatória, tais como, sair da cama, vibração pulmonar e drenagem de secreção também podem evitar a infecção pulmonar e a trombose venosa de membros inferiores.

Concluindo, esta experiência pode oferecer uma solução para esses pacientes. Entretanto, ainda são necessários estudos conduzidos com grandes amostras para se encontrar o melhor tratamento.

\section{Referências}

1. Ross J Jr, Braunwald E. Aortic Stenosis. Circulation. 1968;38(1 Suppl):61-7. doi: 10.1161/01.cir.38.1s5.v-61.

2. Caetano F, Almeida I, Seca L, Botelho A, Mota P, Marques AL. Severe Aortic Stenosis and Cardiogenic Shock: A Therapeutic Challenge. Rev Port Cardiol. 2013;32(9):701-6. doi: 10.1016/j.repc.2012.12.011.

3. Vahanian A, Alfieri O, Andreotti F, Antunes MJ, Barón-Esquivias G, Baumgartner $\mathrm{H}$, et al. Guidelines on the Management of Valvular Heart Disease (version 2012): the Joint Task Force on the Management of Valvular Heart Disease of the European Society of Cardiology (ESC) and the European Association for Cardio-Thoracic Surgery (EACTS). Eur J Cardiothorac Surg. 2012;42(4):1-44. doi: 10.1093/ejcts/ezs455.

4. Leon MB, Smith CR, Mack M, Miller DC, Moses JW, Svensson LG, et al. Transcatheter Aortic-Valve Implantation for Aortic Stenosis in pAtients who Cannot Undergo Surgery. N Engl J Med. 2010;363(17):1597-607. doi: 10.1056/NEJMoa1008232.

5. Smith CR, Leon MB, Mack MJ, Miller DC, Moses JW, Svensson LG, et al. Transcatheter Versus Surgical Aortic-Valve Replacement in High-Risk Patients. N Engl J Med. 2011;364(23):2187-98. doi: 10.1056/NEJMoa1103510.

6. Leon MB, Smith CR, Mack MJ, Makkar RR, Svensson LG, Kodali SK, et al. Transcatheter or Surgical Aortic-Valve Replacement in Intermediate-Risk Patients. N Engl J Med. 2016;374(17):1609-20. doi: 10.1056/NEJMoa1514616.

\section{Conclusão}

O TAVI assistido por ECMO pode ser uma escolha razoável para pacientes com estenose aórtica grave pré-operatório complicada por FE baixo, insuficiência cardíaca, ou até mesmo choque cardiogênico. Por enquanto, um controle pós-operatório razoável pode evitar efetivamente complicações relacionadas ao ECMO e melhorar o prognóstico dos pacientes.

\section{Contribuição dos autores}

Concepção e desenho da pesquisa: Zhang W, He F; Obtenção de dados e Análise e interpretação dos dados: Chen H; Análise estatística: Huang G; Obtenção de financiamento: Chen $\mathrm{H}$, Zhang W; Redação do manuscrito: Huang G; Revisão crítica do manuscrito quanto ao conteúdo intelectual importante: He F.

\section{Potencial conflito de interesse}

Não há conflito com o presente artigo

\section{Fontes de financiamento}

O presente estudo foi financiado por China International Medical Foundation (CIMF) (No. CIMF-Z-2016-23-1823).

\section{Vinculação acadêmica}

Não há vinculação deste estudo a programas de pósgraduação.

\section{Aprovação ética e consentimento informado}

Este artigo não contém estudos com humanos ou animais realizados por nenhum dos autores.
7. Baumgartner H, Falk V, Bax JJ, De Bonis M, Hamm C, Holm PJ, et al. 2017 ESC/EACTS Guidelines for the Management of Valvular Heart Disease. Eur Heart J. 2017;38(36):2739-91. doi: 10.1093/eurheartj/ehx391.

8. Huang J, Chen P, Hu X, Tang J, Fang Z. Aortic Stenosis Complicated by Cardiogenic Shock Treated by Transcatheter Aortic Valve Replacement With Extracorporeal Membrane Oxygenation: A Case Report. Medicine. 2018;97(33):e11900. doi: 10.1097/MD.0000000000011900.

9. Abrams D, Combes A, Brodie D. Extracorporeal Membrane Oxygenation in Cardiopulmonary Disease In Adults. J Am Coll Cardiol. 2014;63(25 Pt A):2769-78. doi: 10.1016/j.jacc.2014.03.046.

10. Annabi MS, Clisson M, Clavel MA, Pibarot P. Workup and Management of Patients with Paradoxical Low-Flow, Low-Gradient Aortic Stenosis. Curr Treat Options Cardiovasc Med. 2018;20(6):49. doi: 10.1007/s11936-018-0642-y.

11. D’Ancona G, Pasic M, Buz S, Drews T, Dreysse S, Kukucka M, et al. Transapical Transcatheter Aortic Valve Replacement in Patients With Cardiogenic Shock. Interact Cardiovasc Thorac Surg. 2012;14(4):426-30. doi: 10.1093/icvts/ivr095.

12. Cheng R, Hachamovitch R, Kittleson M, Patel J, Arabia F, Moriguchi J, et al. Complications of Extracorporeal Membrane Oxygenation for Treatment of Cardiogenic Shock and Cardiac Arrest: A Meta-Analysis of 1,866 Adult Patients. Ann Thorac Surg. 2014;97(2):610-6. doi: 10.1016/j.athoracsur.2013.09.008. 